\title{
Direct oral anticoagulants in atrial fibrillation: can data from randomized clinical trials be safely transferred to the general population? No
}

\author{
Marco Marietta ${ }^{1}$
}

Received: 7 June 2015/ Accepted: 22 June 2015/Published online: 14 July 2015

(C) SIMI 2015

\begin{abstract}
Direct oral anticoagulants (DOAC) represent an innovative and relevant treatment for the prevention of cardiac embolism in patients with atrial fibrillation (AF). Their introduction has been followed by an ample debate on their appropriate use, considering that they can offer an effective treatment for the many patients with AF, which are not taking any effective anticoagulant treatment, even though they have a substantial thromboembolic risk (1). On the other hand, DOAC are much less tested in everyday clinical practice and much more expensive than anti-vitamin $\mathrm{k}$ anticoagulants (AVKs). Starting from the quite favorable results of the available randomized controlled trials (RCTs) - showing that DOAC are at least non-inferior to AVK and that may be even better for some outcomes-this article discusses their transferability to the majority of AF patients. In summary, the body of evidence supports the efficacy and safety of DOAC in patients carrying demographic and clinical characteristics similar to subjects included in RCT, but their use in less well-characterized subpopulations requires particular caution, while waiting for more reliable data from the real world.
\end{abstract}

Keywords Apixaban - Dabigatran - Rivaroxaban - Direct oral anticoagulants · Warfarin · Anti-vitamin k anticoagulants

Marco Marietta

marco.marietta@unimore.it

1 Dipartimento Integrato di Oncologia, Ematologia e Patologie dell' Apparto Respiratorio U.O.C. di Ematologia, Azienda Ospedaliero-Universitaria Policlinico di Modena, Ospedale Policlinico, via del Pozzo 71, 41100 Modena, Italy
Direct oral anticoagulants (DOAC) represent an innovative and relevant treatment for the prevention of cardiac embolism in patients with atrial fibrillation (AF).

Their introduction has been followed by an ample debate on their appropriate use, considering that they can offer an effective treatment for many patients with AF, who are not taking any effective anticoagulant treatment, even though they have a substantial thromboembolic risk [1]. On the other hand, DOAC are much less tested in everyday clinical practice and much more expensive than anti-vitamin $\mathrm{k}$ anticoagulants (AVKs).

DOAC have been extensively evaluated in comparison to warfarin for the prevention of cardiac embolism in patients with non-valvular AF in several randomized controlled trials (RCTs) of good internal methodological quality [2-4]. All these studied were designed as non-inferiority trials, and if non-inferiority was attained, the results were analyzed for superiority. Their results have been pooled in many, well-conducted meta-analyses; moreover, several indirect treatment analyses unfruitfully attempt to demonstrate an advantage from the use of one particular DOAC with respect to another $[5,6]$.

Overall, DOAC have been proved to reduce stroke or systemic embolic events compared with warfarin and allcause mortality, mainly because of reductions in hemorrhagic stroke and intracranial hemorrhage; on the other hand, DOAC significantly increases gastrointestinal bleeding [6].

On these grounds, it could be thought that DOAC are the first and almost unique choice for all patients with $\mathrm{AF}$ and a substantial thromboembolic risk.

However, before drawing such a conclusion, it is crucial to understand whether the good results of the above-mentioned RCT can be generalised to the entire population of AF patients, and I think that the answer to such a compelling question is: "no" for many reasons. 
The strict selection criteria of RCT led to the exclusion of many patients frequently encountered in real-life clinical practice, such as those with impaired renal function (i.e., creatinine clearance $<30 \mathrm{ml} / \mathrm{min}$ ), who represent up to $15 \%$ of the patients attending medical departments. Moreover, it is known that a large proportion of elderly AF patients have severe or moderate renal impairment, because renal function declines with age and a creatinine clearance reduction of about $1 \mathrm{ml} / \mathrm{min} /$ year after the age of 40 is estimated. [7].

The topic of age deserves some further considerations. Although the mean age of patients included in RTC evaluating DOAC is similar to those reported in observational studies performed in routine practice, special attention must be paid to the very older patients, i.e., those aged $>80$ years [8]. Very few data are available about this peculiar group of frail patients, because the RCTs report a percentage of patients aged $\geq 75$ years, ranging between 31 and $43 \%$, but they do not further stratify the older ones, who represent about one-third of patients receiving AVK for $\mathrm{AF}$ in the clinical practice [8].

A recent meta-analysis demonstrates that in participants of clinical trials aged $>75$ years NOAC do not cause excess bleeding, and are associated with equal or greater efficacy than conventional therapy [9].

Although this finding seems reassuring, it does not fully answer the question about the safety and the efficacy of DOAC in very elderly patients, such as those aged 80 and older. Relevant to this, a post hoc analysis of the RE-LY study demonstrated that the risk of major bleeding in patients treated with Dabigatran was correlated with the through concentration of the drug, which in turn depended on the age of the subjects [10]. The same study demonstrates that the risk of major bleeding is increased, at the same Dabigatran plasma levels, in subjects older than 85 years as compared with those of 75 years. This finding is confirmed by a recent study, which evaluated the safety of dabigatran versus warfarin for treatment of non-valvular atrial fibrillation in general practice settings [11]. This study finds an increased risk of major gastrointestinal bleeding with dabigatran in women aged $\geq 75$ years and in men aged $\geq 85$ years. Moreover, the same study demonstrates a shift in hazard ratio between younger and older women, suggesting that the benefit/risk profile of dabigatran may be less favorable in women aged $\geq 85$ years than in other age/sex groups [11].

On these premises, one can expect a cautious use of DOAC among the oldest patients in clinical practice, whereas it has been demonstrated that prescription rates of dabigatran are most accelerated, at least initially since its approval, among patients aged 85 or older. Although AVKs also carry an increased risk of major bleeding when given to very elderly patients, their use in such population is validated by a more than fifty years long experience and by clinical trials demonstrating their good efficacy and safety in such a peculiar clinical context [12].

Therefore, I suggest that while also expecting data confirming their safety in this population, DOAC should be cautiously used in patients aged 80 years and older, and after having excluded the possibility of a well-managed therapy by AVKs.

The careful selection on clinical grounds of the population included in the registration trials of DOAC contrasts with the heterogeneity of the same population with regard to the quality of the treatment obtained with by the comparator drug, i.e., warfarin. Indeed, the mean Time in Therapeutic Range [TTR] of the trial arms receiving warfarin ranges from 55 to $68 \%$, which is less than the $76.5 \%$ that can be attained by well-trained centres, as recently reported by the Swedish national anticoagulation register Auricula [10]. For instance, the value of TTR recorded in the centres participating in the RE-LY trial is very scattered, ranging from $44 \%$ observed in Taiwan or India to $80 \%$ recorded in the Sweden [2]. This point deserves attention, as the overall benefits of DOAC in terms of efficacy and safety are attenuated or lost at centres and in patients with predicted excellent INR control [13-15].

Another point deserving attention is the concomitant use of antiplatelet (APL) and oral anticoagulants, either AVK or DOAC. This is frequently found [16].

Very few data are available from prospective studies on the combined use of APL with AVK or DOAC, and we have to turn to post hoc analyses from registration trials to obtain a better understanding of the benefits and risk of adding APL to a DOAC. In these trials, concomitant aspirin or $\mathrm{P}_{2} \mathrm{Y}_{12}$ receptor antagonist use was left to the discretion of the treating physician, whereas patients requiring double antiplatelet treatment at baseline were not eligible.

As far as the RELY trial is concerned, only one in 5 patients used APL continuously throughout the study (median duration, $66 \%$ of the total study duration); aspirin was by far the antiplatelet agent most often used, whereas the use of clopidogrel only $(1.9 \%)$ was infrequent. Other $\mathrm{P}_{2} \mathrm{Y}_{12}$ inhibitors such as ticagrelor or prasugrel were not used. In the ROCKET trial, $34 \%$ of patients were taking APL "at some time during the study," but no data are available about the proportion of patients who continuously used both drugs. Finally, about $20 \%$ of patients included in the ARISTOTLE trial concomitantly used APL and apixaban over the entire time of the study. Relevant to this, it must be observed that the allowed dose of aspirin varied among the trials from less than $100 \mathrm{mg}$ in RELY and ROCKET to $165 \mathrm{mg}$ in the ARISTOTLE trial.

Overall, the concomitant use of aspirin and DOAC, while increasing the bleeding risk, does not seem to affect 
the advantages of these drugs over warfarin, but the analyses are based on the use of APL at some time during the median 2-year treatment period. Therefore, the risk associated with use for longer times may be higher.

Moreover, no data are available about the bleeding risk in patients taking a "triple therapy" which represent a small but significant part of AF patients. In such patients, it would be advisable to prefer warfarin over DOAC, because of the virtual absence of data about the latter, as compared with a well-established experience with the former.

Perioperative management of DOAC is another open issue [17]. Sub-analyses of the registration trials of DOAC involving patients who underwent invasive procedures have been published, as well as algorithms for management with new DOAC around the time of surgery [17].

Overall, low rates of complications are found and fatal complications seem to be very rare, indicating that periinterventional short-term interruption of DOAC can be regarded as safe in most cases. However, such analyses carry limitations because of their retrospective nature, and prospective studies are needed to assess the safety and efficacy of standardized perioperative management strategies involving patients who are receiving DOAC and require an elective or urgent surgery or procedure. Moreover, the question of whether, and when, using perioperative bridging with low-molecular heparin in patients receiving DOAC remains unanswered, whereas such a strategy is widely used in patients receiving AVK, despite the weakness of the evidence about its efficacy and safety.

Finally, the patients enrolled within RE-LY and ARISTOTLE trials were quite different as far as the thromboembolic risk is concerned in comparison to those of ROCKET trial [2-4]. Relevant to this, it has been found that patients enrolled within the former two trials were more reflective of the 'real-world' AF population in the $\mathrm{UK}$, in contrast with patients included in the ROCKET-AF trial, who represent a group of patients at higher risk of stroke. Differences between trials should be taken into account when considering the applicability of findings from RCTs, although assessing representativeness it is not a substitute for assessing generalizability.

In order to do that, the external validity of the phase III RCT needs to be confirmed in the daily care setting, where patients may have significant comorbidities and are treated without a strict protocol and under less intense surveillance. However, undertaking such real-life assessments typically takes several years, as the drugs in question need to become used widely.

Some data from the real-world use of DOAC appeared on the medical literature, but the findings are not unequivocal and sometimes even confounding, perhaps because of the different populations evaluated as well as of the different methods used for collecting data [18-20].
Overall, these data seem to confirm the efficacy and safety profile showed by DOAC in registration trials, but a few comments are required.

The majority of data about the daily use of DOAC regard dabigatran, and to a lesser extent rivaroxaban, whereas no data have been published so far about the realworld use of apixaban. [18-20]. As far as dabigatran is concerned, two studies, despite their different design, show an excess of bleeding in patients receiving the $110 \mathrm{mg}$ BID dose $[18,19]$. Interestingly, in both cases patients receiving this dose were significantly older than those receiving the higher one (respectively, 79.6 vs 69.7 and 78 vs 71 years). Moreover, both studies showed an increased risk of any bleeding in patients who switched to dabigatran compared with those who remained on AVK.

In my opinion, these findings suggest the need for particular caution in prescribing DOAC to the very elderly patients; moreover, in this group of patients, it would be advisable to limit the shift to DOAC from AVK to those who have an unsatisfactory TTR.

In summary, the body of evidence supports the efficacy and safety of DOAC in patients carrying demographic and clinical characteristics similar to subjects included in RCT, but their use in less well-characterized subpopulations requires particular caution, while waiting for more reliable data from the real world.

\section{Compliance with ethical standards}

Conflict of interest The authors declare that they have no conflict of interest.

Statement of human and animal rights All procedures performed in studies involving human participants were in accordance with the ethical standards of the institutional and/or national research committee and with the 1964 Helsinki declaration and its later amendments or comparable ethical standards. This article does not contain any studies with human and animals performed by any of the authors.

Informed consent None.

\section{References}

1. Palareti G, Salomone L, Cavazza M, Guidi M, Muscari A, Boriani G et al (2014) Stroke/thromboembolism and intracranial hemorrhage in a real-world atrial fibrillation populationcomplications in patients with atrial fibrillation: the complications of atrial fibrillation in the bologna area (CAFBO) study. Chest 146:1073-1080

2. Connolly SJ, Ezekowitz MD, Yusuf S, On the behalf of the RELY Steering Committee and Investigators et al (2009) Dabigatran versus warfarin in patients with atrial fibrillation. N Engl J Med 361:1139-1151

3. Patel MR, Mahaffey KW, Garg J, On behalf of the ROCKET AF Investigators et al (2011) Rivaroxaban versus warfarin in nonvalvular atrial fibrillation. N Engl J Med 365:883-891

4. Granger CB, Alexander JH, McMurray JJ, On the behalf of ARISTOTLE Committees and Investigators et al (2011) 
Apixaban versus warfarin in patients with atrial fibrillation. N Engl J Med 365:981-992

5. Dentali F, Riva N, Crowther M, Turpie AG, Lip GY, Ageno W (2012) Efficacy and safety of the novel oral anticoagulants in atrial fibrillation: a systematic review and meta-analysis of the literature. Circulation 126:2381-2391

6. Ruff CT, Giugliano RP, Braunwald E, Hoffman EB, Deenadayalu N, Ezekowitz MD et al (2014) Comparison of the efficacy and safety of new oral anticoagulants with warfarin in patients with atrial fibrillation: a meta-analysis of randomised trials. Lancet 383:955-962

7. Poli D, Antonucci E, Zanazzi M, Grifoni E, Testa S, Ageno W, Palareti G (2012) Impact of glomerular filtration estimate on bleeding risk in very old patients treated with VKA: results of EPICA study on the behalf of FCSA (Italian Federation of Anticoagulation Clinics). Thromb Haemost 107:1100-1106

8. Sjögren V, Grzymala-Lubanski B, Renlund H, Friberg L, Lip GYH, Svensson J, Själander A (2015) Safety and efficacy of well managed warfarin; a report from the Swedish quality register Auricula. Thromb Haemost 26(113):1370-1377

9. Sardar P, Chatterjee S, Chaudhari S, Lip GY (2014) New oral anticoagulants in elderly adults: evidence from a meta-analysis of randomized trials. J Am Geriatr Soc 62:857-864

10. Reilly PA, Lehr T, Haertter A, On behalf of the RE-LY Investigators et al (2014) The effect of dabigatran plasma concentrations and patient characteristics on the frequency of ischemic stroke and major bleeding in atrial fibrillation patients. The RELY trial (randomized evaluation of long-term anticoagulation therapy). J Am Coll Cardiol 63:321-328

11. Graham DJ, Reichman ME, Wernecke M, Zhang R, Southworth MR, Levenson M et al (2015) Cardiovascular, bleeding, and mortality risks in elderly medicare patients treated with dabigatran or warfarin for nonvalvular atrial fibrillation. Circulation 131:157-164

12. Poli D, Antonucci E, Testa S, On behalf of Italian Federation of Anticoagulation Clinics et al (2011) Bleeding risk in very old patients on vitamin $\mathrm{K}$ antagonist treatment: results of a prospective collaborative study on elderly patients followed by Italian Centres for Anticoagulation. Circulation 124:824-829

13. Wallentin L, Yusuf S, Ezekowitz Md, On behalf of the RE-LY investigators et al (2010) Efficacy and safety of dabigatran compared with warfarin at different levels of international normalised ratio control for stroke prevention in atrial fibrillation: an analysis of the RE-LY trial. Lancet 376:975-983

14. Wallentin L, Lopes RD, Hanna M, Thomas L, Hellkamp A, Nepal S et al (2013) Efficacy and safety of apixaban compared with warfarin at different levels of predicted international normalized ratio control for stroke prevention in atrial fibrillation. Circulation 127:2166-2176

15. Piccini JP, Hellkamp AS, Lokhnygina Y, Patel MR, Harrell FE, Singer DE et al (2014) Relationship between time in therapeutic range and comparative treatment effect of rivaroxaban and warfarin: results from the ROCKET AF Trial. J Am Heart Assoc 3:e000521

16. Rubboli A, Oldgren J, Marìn F, Lip G (2013) Combination of a new oral anticoagulant, aspirin and clopidogrel after acute coronary syndrome: new therapeutic standard? Intern Emerg Med 8:673-680

17. Liew A, Douketis J (2013) Perioperative management of patients who are receiving a novel oral anticoagulant. Intern Emerg Med 8:477-484

18. Sørensen R, Gislason G, Torp-Pedersen C, Olesen JB, Fosbøl EL, Hvidtfeldt MW et al (2013) Dabigatran use in Danish atrial fibrillation patients in 2011: a nationwide study. BMJ Open 3:e002758

19. Beyer-Westendorf J, Ebertz F, Förster K, Gelbricht V, Michalski F, Köhler C et al (2015) Effectiveness and safety of dabigatran therapy in daily-care patients with atrial fibrillation. Results from the Dresden NOAC registry. Thromb Haemost 113:1247-1257

20. Tamayo S, Peacock WF, Patel M, Sicignano N, Hopf KP et al (2015) Characterizing major bleeding in patients with nonvalvular atrial fibrillation: a pharmacovigilance Study of 27467 patients taking rivaroxaban. Clin Cardiol 38:63-68 